notable for their absence among women with subarachnoid haemorrhage and their controls. As in most series, the number of individuals with myocardial infarction, and especially with subarachnoid haemorrhage, is very small. We hope that the authors will be able to evaluate a case series of sufficient size to address the concern that current use, particularly of highpotency or high-dose oestrogens, may have grave effects, such as those described in olde studies on prostate cancer ${ }^{4} 5$ and secondary prevention of coronary heart disease. ${ }^{6}$

ROBERT I PFEFFER

Department of Neurology

California College of Medicine,

Orange, California 92668,

USA

' Pfeffer RI, van den Noort S. Am $\mathcal{Y}$ Epidemiol 1976; 103:445-56.

Pfeffer RI. $\mathcal{F}$ Chron Dis 1978 ;81:389-98.

Pfeffer RI, Whipple GH, Kurosaki TT, et al. Am $\mathcal{J}$

Epidemiol 1978;107:479-87.

$1970 ; 26: 249-56$.

s Byar DP. Cancer 1973;32:1126-30

Coronary Drug Project Research Group. JAMA $1973 ; 226$ :652-7.

\section{Pertussis vaccine}

SIR,-Your leading article on pertussis vaccine (16 May, p 1563) raises points of importance for the future of pertussis immunisation itself and for drug and vaccine safety in general. The comments which follow are based on persona views I have formed as chairman of the Committee on Safety of Medicines' (CSM's) Advisory Panel on the Collection of Data Relating to Adverse Reactions to Pertussis Vaccine. ${ }^{1}$ This was set up in 1977 to assess data submitted by the Association of Parents of Vaccine Damaged Children (APVDC) together with the CSM's own data on adverse reactions.

(1) Were the risks exaggerated?-Your leading article, and a rather similar article in The Times of $13 \mathrm{May}$, drew attention to estimates of neurological damage as high as one in 10000 vaccinations. In fact, the papers in question ${ }^{23}$ actually spoke of risks per 10000 children. The estimates were thus less extreme than you implied. A summary of the main estimates for the $U K$ is shown in the accompanying table. All have wide margins of uncertainty and their interpretation is subject to a number of qualifications. Firstly, the figures depend heavily on definitions. One which includes convulsions not followed by other events will give a much higher figure than one restricted to irreversible brain damage. The latter is the outcome on which the figures most widely quoted in the last few weeks have been based. However, recovery from a serious neurological illness is certain only in retrospect. Some attention must therefore be paid to the risk of such illnesses, as well as to that of irreversible damage.

Secondly, risks may have been higher before 1974-5 than after. It seems probable (see below) that contraindications to pertussis vaccine were often overlooked before 1974-5. If they have been more closely observed since risks may have been less. Absolute numbers of children affected over a given time were probably larger before 1974-5, mainly because pertussis vaccine uptake rates were then about $80 \%$ rather than the subsequent $30 \%$ but also because of the higher birth rate. (Your leading article states that two children out of 600000 given pertussis vaccine will have permanent disability. Should not the figure have been six ?)

Thirdly, our panel's estimate is tentative, for reasons given in the report. Finally, the National Childhood Encephalopathy Study (NCES) ${ }^{14}$ has shown that its estimates are especially sensitive to possible underascertainment of cases. Our panel found that about a third of the serious APVDC and CSM cases had not been admitted to hospital and would thus have been missed by the NCES (on the assumption that patterns of admission in 1970-4 are applicable to 1976-9). It thus seems reasonable to present the two sets of estimates. The children not admitted to hospital in our series were by no means confined to those with infantile spasms. In the table the NCES figures are expressed in terms of children immunised rather than the number of immunisations given, a course also adopted by the Joint Committee on Vaccination and Immunisation (JCVI). ${ }^{1}$ The original reports should be consulted for exact definitions, further qualitications, and the effects of possible underascertainment of cases on estimates other than those from the NCES.

(2) Was the safety of pertussis vaccine exaggerated? -The JCVI report ${ }^{5}$ of 1977 may have given others besides myself the impression of a degree of reassurance that was not justified by the information then available. What cannot be disputed is that the report referred to a risk of "brain damage" of one in 300000 . That the figure is now generally regarded as misleading does not alter the fact that it was widely cited at the time. ${ }^{6}$ The Evening Standard of 4 October 1979, under a heading "Whooping cough vaccine gets all-clear," gave an almost categorical assurance of safety based on a presentation of Public Health Laboratory Service (PHLS) data to the Royal Institute of Public Health: "Not a single case of brain damage. . Most doctors now accept the vaccine is safe." I am not aware of any attempt by the DHSS or PHLS to modify the newspaper report. Our panel was not allowed to see up-to-date PHLS data. (The proffered explanation-their incompleteness-did not prevent the data being presented to others dealing with the pertussis question.)

Views on safety may also have been influenced by your account of the 35 NCES children vaccinated within seven days. You reduce these to five children, previously normal, with serious neurological damage and no explanation other than the diphtheria, tetanus, and pertussis vaccine. But a case-control study is intended to establish relative risks, not to make diagnoses in individual cases. If there are to be exclusions, they should be made before not after the study. In any case, the vaccine may have unmasked or aggravated other illnesses, as the NCES itself points out. ${ }^{1}$ (The NCES makes it clear that its attributable risk figures are based on all the vaccine-associated cases.)

(3) Contraindications-Our panel was struck by the number of cases in which immunisations had been carried out despite contraindications. About half the children in our series were given at least one injection that was contraindicated. In a quarter of these cases the contraindication was a reaction to a preceding injection. There were two previously normal children in whom grand-mal convulsion occurred within 72 hours of each of two successive injections. One died after years of intractable epilepsy. The other developed immediate evidence of brain damage after the second injection and became grossly mentally retarded. These are extreme but salutary examples. We do not know how many children with contraindications were immunised without mishap. We could not absolutely exclude the possibility that some of the affected children were immunised after full account had been taken of possible contraindications. We do not claim with certainty that pertussis vaccine was the cause of subsequent events in all the children. But the case histories provided no justification for treating contraindications as "myths."

If we take the NCES figures as the yardstick, it is doubtful whether the risks of pertussis immunis-

Estimates of frequency of events in immunised children

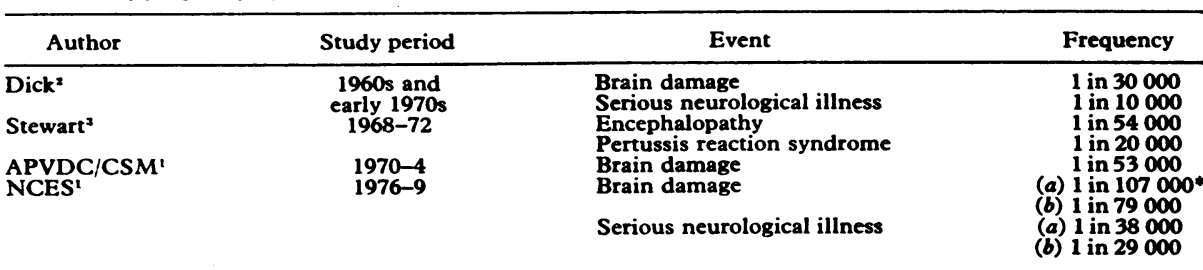

* NCES data shown (a) without adjustment; (b) with adjustment for possible underascertainment.

APVDC = Association of Parents of Vaccine Damaged Children; CSM=Committee on Safety of Medicines; NCES $=$ National Childhood Encephalopathy Study. ation were exaggerated to the extent usually suggested. I do not accept all Professor Gordon Stewart's arguments for a cause- und-effect relationship or for a pertussis reaction syndrome. Nor do I share Professor Stewart's reservations on the value pertussis vaccine. His contribution towards ensuring that we now have at least some of the facts about its risks must, however, be acknowledged. Is he the only person to have identified an adverse effect without being able precisely to quantify it early on ? Whose responsibility is it that there was no systematic information when the question was first raised? If there was any exaggeration of the risks it was accompanied by an exaggeration of safety. Public anxiety was "fuelled," as you put it, not only by warnings about risks but also by the fact that there was quite evidently no firm basis for the reassurances that were given.

As far as pertussis immunisation is concerned, the past has two lessons for the future. Firstly, restoration of public confidence in the programme will be helped rather than hindered by a frank recognition of the difficulties faced by those, especially parents, who have to make decisions for individual children. Secondly, systematic methods for identifying contraindications and for deciding how to proceed when they are present should be

More generally, the pertussis vaccine episode is another example of the short comings of present methods for monitoring the safety of drugs and vaccines. Until there are improvements, we should be very careful about talking of alarmism. Some scares may turn out to be groundless-but others may not. Last year, at a time when he may well have known the outcome of the pertussis studies, the Minister of State for Health turned down a CSM proposal which would have been a first step towards a better system for studying adverse drug effects. Vaccines could have been included in later extensions of the system. Some of the CSM's proposals may be carried out through other channels. But the failure of even the pertussis episode to move the Government towards strengthening its own drug and vaccine safety organisations is regrettable.

T W MEADE

\section{Medical Research Council \\ Epidemiology and Medical \\ Care Unit, \\ Northwick Park Hospital,}

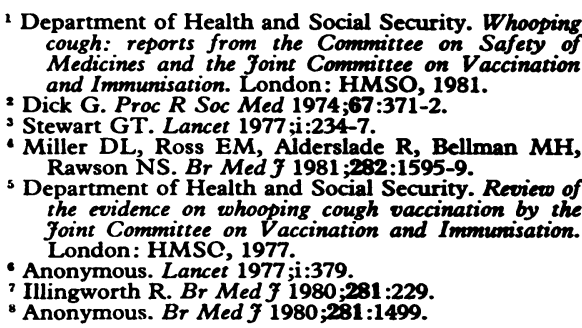

* * What are the implications of a risk of one in 300000 immunisations for the standard immunisation programme? The answer depends on the likelihood that an infant who did 
not react adversely to a first or second injection might develop permanent brain damage from a reaction to the third. Such a response is, we believe, unlikely; but until that question is answered our estimate of two victims a year is as plausible as Dr Meade's suggestion that the number might be six.-ED, $B M F$.

\section{Effect of vaccination on severity and dissemination of whooping cough}

SIR,-Dr P R Grob and others (13 June, p 1925) have shown that "whooping cough vaccination modifies the clinical illness" by shortening its duration and reducing the severity of cough and incidence of complications, "and offers a worthwhile degree of protection to children exposed to the disease." But is modification of the severity of an illness by immunisation an unmixed blessing ?

In Sheffield in $1887^{1}$ and Brighton in $1950^{2}$ modified smallpox in vaccinated persons was responsible for the unsuspected spread of infection in the first stages of both epidemics. Modified cases may be individually less dangerous than the unmodified but they are more often in ambulant people and go unrecognised and uncontrolled.

There is a difference also between complications such as pneumonia and neurological complications. Pneumonia, bronchitis, and most other dangerous consequences are related to the severity of the original infection; but neurological complications are not. In varicella encephalitis there is no relation between its severity and the severity of the preceding rash. Measles encephalitis has several times been reported after the mild illness which follows attenuating doses of gammaglobulin. ${ }^{3}$

How do we know that modified cases of whooping cough, missed or even subclinical, are not actively spreading infection, or that a substantial number of apparently primary cases of encephalitis are not, in reality, complications of half-prevented disease?

\section{P E BRown}

Department of Community Medicine, Medical School,
Sheffield S10 2RX

\section{Report on smallpox at Sheffield during 1887-88. London: HMSO, 1889:4-5. \\ Leff S. Recent outbreaks of infectious diseases. London: Lewis, 1957:64-77. Miller HG, Stanton JB, Gibbons JL. Q f Med 1956;
25:427-505.}

\section{Brain death in three neurosurgical units}

SIR,-Dr E W Poole (18 April, p 1316) asks very pertinently for more details of the neurosurgical data base on which was constructed part of the paper seeking to validate the UK Royal Colleges' brain death criteria (14 February, p 533). He will appreciate the demands of concision and realise that to have presented all the information available for 609 patients would have tended to make a dauntingly long and unduly anecdotal article. After all, "Death has a thousand doors to let out life." I will try, however, to answer some of Dr Poole's questions, confining myself to the 124 Swansea cases of brain death with ventilation.

Ages-The head-injured group (54 patients) comprised 10 children (0-15 years), 19 young adults (15-30 years), six middle-aged patients (30-50 years), and 19 elderly patients (over 50 years). Children and young adults thus made up $54 \%$ of this category. By contrast, the intracranial haemorrhage group (51 patients) contained only two children and no young adults. Similarly, "Other conditions" (encephalitis, brain tumours abscess, infarction, anoxic encephalomalacia19 patients in all) included no children and only two young adults.

The diagnostic base-One hundred and twentytwo patients $(98 \%)$ had at least one computed tomography (CT) scan. Fifty patients $(40 \%)$ had at least one cranial operation with therapeutic intent. Ten patients, none of them head injured, had had diagnostic cerebral angiography. There were no instances even among the acute head injuries where drugs, including alcohol, played any determinant role in either the coma or its outcome. Hypoxia complicating primary head injury, however, was an important-probably decisive-facto in a handful of patients. It was occasioned by airway obstruction before admission in three cases and by acute pulmonary oedema in two cases. There was only one instance of pure anoxic encephalomalacia. This followed surgical and anaesthetic misadventure during nephrectomy, with survival for nearly 44 days before stem reflexes petered out. No primary metabolic disorders were encount :red, though many secondary metabolic (or catabolic) problems supervened in the longer survivors. One elderly ventilated patien never regained consciousness or ability to breathe after craniotomy for a large suprasellar tumour, but probably died from the effects of subsequent frank myocardial infarction. The relative roles of his cranial and cardiac conditions were open to debate.

Pathology-All dying from head injury had a statutory necropsy. In each was found precise correlation between morbid anatomy and CT scan. (One head injured man was never scanned because of an associated high cervical dislocation.) Fifty-two patients had severe diffuse brain contusions or lacerations, or both, usually with basal skull fracturing. In two cases the primary brain injury had been accompanied by relevant extradural haemorrhage. In a further two extradura haemorrhage had been the essential lesion. All clots had been surgically removed. Two patients also had ruptured spleen and haemoperitoneum one treated in life and the other undiagnosed till necropsy. In the two other broad categories, a least one CT scan had been performed in all bu one instance. Necropsy was done in $80 \%$; in the exceptions relatives' permission was withheld. Again, a precise correlation of postmortem findings with CT data was found. No unexpected dis coveries were made.

In the spontaneous haemorrhage group, $57 \%$ died from massive aneurysmal bleeding. One of these had rebled from a previously treated (wrapped) aneurysm. There were three deaths from progressive cerebral infarction after elective aneurysm surgery. Three patients died from angioma haemorrhage. The remainder of the group bled from "medical" causes, including hypertension. Of five instances of fulminating herpes encephalitis, four were proved virologically during life, and on not until after death. Of the 10 brain tumour patients, eight had undergone some form of internal decompressive surgery. Five of the eight had histologically benign tumours. The two unoperated patients had advanced gliomas. One died of an associated haemorrhage and one of a supervening middle cerebral occlusion.

How did the signs of brain death evolve?-Usually signs of brain death developed all at once-most conspicuously so in the severely head injured and those with massive destructive haemorrhages and haemocephalus. But in several patients with longer time courses caloric reflexes were noted to flicker on until some hours before spontaneous heart stop.

When was "absolute predictive certainty" established?-To pin down the curiously elusive variable of absolute predictive certainty it has seemed to me most apt to examine the small bu relatively homogeneous group of 25 transplant donors.

Head injured donors had been in hospital for 14-138 hours (average 55 hours) and ventilated for 8-124 hours (average 46). Time elapsing between definitive clinical diagnosis and the actua cardiac end point varied from 2 and 30 hours (average 9 hours). Brain haemorrhage donors had been in hospital for 24-624 hours (average 187 hours) and ventilated for an average of 56 hours. The interval from brain death to the cardiac end point averaged 10 hours.

"Absolute predictive certainty" was established earlier in those hospitalised for shorter times, simply because these were patients with the more devastating injuries and the more catastrophic bleeds. The shortest time between neurosurgical admission and the point of predictive certainty was three hours. This was in a badly head injured man who went on to spontaneous heart stop. The longest interval, also in a patient with a gross compound head injury, was close on 100 days of what Dr Poole might call "enthusiastic maintenance."

The natural history of survival-I am unsure what Dr Poole means by this term. Nothing in the milieu of neurosurgical intensive care is natural. But in the period 1977-80, when 105 ventilated patients with head injury and spontaneous haemorrhage died, there were 26 who survived ventilation and left hospital-that is, $20 \%$ of all those in the two categories reviewed. A further 19 "miscellaneous" patients survived elective ventilation and made good recoveries. These were patients with overdose, eclampsia, encephalitis, cerebellar abscess (one each), myasthenia, various forms of polyneuritis, acute shunt dysfunction, and postcraniotomy problems. In no survivor were the brain death criteria at any juncture fulfilled. In most it was inappropriate to apply them.

I agree with Dr Poole that, reassuring as retrospective analysis has been-there were no "near misses"-prospective studies with investigative correlations should not on that account be neglected. His most important point has been made many times before. ${ }^{23}$ It cannot be made too often or emphasised too much. It is this. Brain death must never be considered unless there is an unshakeable basic diagnosis of destructive brain disorder. Granted that irreducible prerequisite, the argument for rigid timings and repetitions of the clinical brain death tests becomes less than compelling-another point already well made. ${ }^{4}$

Department of Surgical

P J E WILSON

Neurology,

Morriston Hospital

\section{Massinger P. A very woman. Act 5, scene 4. 1655. Wilson PJE. Rr Med $尹$ 1980;281:1140. \\ Wilson PJE. Daily Telegraph 1981; March 4 \\ - McGillivray B. Daily Telegraph 1981;March 3.}

\section{Endoscopic assessment of oesophageal} disease

SIR,-Mr K S Mullard's letter (18 April, p 1320) is in further support of his contention that any endoscopic examination for suspected oesophageal disease should be preceded by a barium swallow, of at least a simple one- or two-bolus type, to establish whether there is obstruction which might lead to difficult instrumentation and the danger of perforation. I think that this is quite unnecessary.

For 10 years now all junior staff trained in our unit have been taught that the end-viewing fibreoptic instruments should be introduced under direct vision so that the lower pharynx and the whole length of the gullet are examined during the advance of the instrument. Any obstruction can be seen and our standard practice is that a difficult instrumentation is not persisted with and that no force is used. In other words, every oesophagoscopy is treated as if there is a potential danger-we do not assume that there is no obstruction in any patient.

Since 1974 we have been able to perform 